\title{
The Effect of Prophylactic Intravenous Fluconazole on the Clinical Outcome of Preterm Infants During Hospitalization
}

\author{
Abdollah Jannatdoust ${ }^{1^{*}}$, Vida Imani ${ }^{2}$
}

\begin{abstract}
Objectives: Systemic fungal infection (SFI) is one of the most common causes of mortality, especially in preterm neonates. This fact necessitates the need to pay more attention to the prophylactic role of antifungal therapy in these neonates. The aim of this study was to evaluate the effect of intravenous fluconazole prophylaxis on the clinical outcome of preterm neonates during hospitalization. Materials and Methods: In a randomized, double-blind clinical trial, 93 preterm neonates with birth weight less than $1250 \mathrm{~g}$ without any major congenital anomalies were divided into control and case groups. Gestational week, birth weight, history of antenatal antibiotics and steroids administration and central venous catheter usage were recorded. During hospitalization, $3 \mathrm{mg} / \mathrm{kg}$ dose of fluconazole was administered for the case group for 6 weeks: in the first 2 weeks every 3 days, in the second 2 weeks every 2 days, and in the third 2 weeks every day; however, the control group did not receive fluconazole. The outcomes, including duration of mechanical ventilation, in-hospital mortality, and duration of oxygen requirement were ultimately recorded. The data from both groups were analyzed using statistical tests and compared to each other.

Results: There were not significant differences in both groups in terms of gestational week of childbirth $(P=.059)$, birth weight $(\mathrm{P}=.342)$ and antenatal steroids administration $(P=.221)$; while duration of mechanical ventilation, duration of hospitalization and the mortality rate were significantly higher in the control group compared with the case group $(P=.002),(P=.011)$ and $(P=.045)$, respectively.

Conclusion: The results show IV fluconazole administration for very low birth weight preterm neonates can improve the prognosis of fungal infection and reduce mortality.

Keywords: Fluconazole, Fungal infections, Preterm infants
\end{abstract}

\section{Introduction}

Despite the great efforts for improving the outcome of preterm neonates, systemic fungal infection (SFI) mainly caused by candida species is a complex part for treatment of neonates with chance of survival. Preterm neonates hospitalized in neonatal intensive care unit (NICU) are strongly at risk for SFIs; this problem can be attributed to unavoidable existence of several risk factors as well as long term, aggressive, and intensive therapies which are done in these patients with incomplete immunization against infective elements (1-5).

Colonization with Candida species, several courses of treatment with antibiotics, especially third-generation cephalosporin, full parenteral nutrition, central venous catheters, gastrointestinal pathology, use of postnatal steroids, use of mechanical ventilation and the use of second subtype of histamine receptor antagonists are risk factors that are most associated with fungal infections, which have been seen in these patients (4-10). Candida species are known as the third factor for infants with very low weight late-onset sepsis (LOS) in preterm neonates with an incidence of $1.6 \%$ to $9 \%$ in and an incidence of $10 \%$ to $16 \%$ in extremely low birth weight neonates (less than $1000 \mathrm{~g}$ ) in NICU; the primary mortality rate is about $30 \%$ to $75 \%$. The mortality rate in these patients is significantly higher compared with infants who did not suffer from a fungal infection (28\% vs $7 \%$ ) (1-5). In fact, $60 \%$ of neonates with birth weight less than $1500 \mathrm{~g}$ during the first month of hospitalization in the NICU are colonized with Candida species due to infection of the skin and oral mucosa that will lead to fungal infection in $20 \%$ of the infants. Therefore, fungal infection increases the number of hospitalization days and the costs of treatment (1-5). These estimates may be reported less than the actual amount because probably fungal infections due to non-specific symptoms, non-permanent isolation of Candida species in blood cultures (sensitivity less than $50 \%$ for the Candida) and low sensitivity of serum inflammatory markers may be diagnosed less than the actual amounts (11). High clinical suspicion and early diagnosis are crucial 
since the delay in the onset of systemic antifungal therapy has been recognized as a cause of increased mortality. For management of SFIs, we need to use the right tool effectively for the early diagnosis and prevention. However, prevention from the risk factors mentioned above in NICU must be considered important before these risk factors lead to infection (12-14).

With regard to the high prevalence of fungal colonization in neonates in NICU and the lack of such a study on the effect of prophylactic intravenous fluconazole on reducing the morbidity and mortality of fungal infections, we have examined the effect of the IV fluconazole on the neonatal outcomes in this study. If this issue proves the effectiveness of this drug in reducing hospital complications, it can be introduced for routine preventive use.

\section{Materials and Methods}

In this randomized, double-blind clinical trial, 100 preterm neonates with birth weight less than $1250 \mathrm{~g}$ were selected from the neonates hospitalized in the NICU of Al-Zahra hospital and Pediatrics hospital, Tabriz, Iran. Then, 7 patients due to major anomalies were excluded from the study. Ultimately, 93 preterm infants with birth weight less than $1250 \mathrm{~g}$ without any major congenital anomalies were divided into 2 groups of control and case. At first, sex, gestational age (week), birth weight, Apgar scores at 1 and 5 minutes, mode of delivery, antenatal use of antibiotics and steroids, and central venous catheter usage were recorded. During hospitalization, the 6-week treatment with $3 \mathrm{mg} / \mathrm{kg}$ dose of fluconazole every 3 days in the first 2 weeks, every 2 days in second 2 weeks and every day in the third 2 weeks was administrated for the case group; however, the control group did not receive fluconazole. Fluconazole prophylaxis was started at the three days of age in the suspected cases of systemic infections, and cultures from blood, urine and cerebrospinal fluid (CSF) were obtained in these cases. Length of hospitalization, the need for mechanical ventilation and the incidence of in-hospital mortality were recorded and compared between the two groups.

Based on a statistical consultant advice and also with regard to similar studies and statistical calculations, total sample size of 100 was sufficient with statistical values for the final report.

Inclusion criteria included birth weight less than $1250 \mathrm{~g}$ and prematurity less (than 32 weeks of gestational age). Meanwhile exclusion criteria included any major congenital anomaly. The variables were:

1. Length of hospitalization

2. Duration of oxygen requirement

3. Antenatal steroids use

4. Birth weight

5. Neonates gender

6. Receiving surfactant

\section{Ethical Considerations}

All the actions taken towards diagnosis and treatment or follow-up of premature infants should be normally per- formed, so it should not impose additional costs on patients. Patient's parents were given insurance that the individuals participation in the study was completely voluntary and confidential as well as their names and addresses would not be mentioned. Integrity and confidentiality of personal information was maintained during the study.

\section{Statistical Analysis}

Descriptive analytic methods (frequency, percentage, mean \pm SD) and comparative methods (the chi-square and mean difference tests were used to analyze the data. All statistical analyses were performed with SPSS 16 statistical software. The $P<.05$ was considered statistically significant in all cases.

\section{Results}

In this study, we studied 93 neonates of which 43 neonates were in the group receiving fluconazole and 50 patients were in the control group. Meanwhile, the neonates were randomly assigned to case (receiving fluconazole) and control groups. These groups showed no significant difference in terms of confounding variables:

- Mean birth weight of infants was $968.83 \pm 163.31 \mathrm{~g}$ in the group receiving fluconazole and $976.38 \pm 203.33$ $\mathrm{g}$ in the control group, which were not significantly different from each other $(P=.342$; Figure 1$)$.

- Mean gestational age of the neonates in the group receiving fluconazole was $28.41 \pm 1.57$ weeks, and the mean gestational age in the control group was $28.76 \pm 2.12$ weeks, the 2 groups did not differ from each other significantly for the birth weeks $(P=.59$; Figure 2).

- In the fluconazole recipient group, 2 patients had received antenatal steroids and 41 patients had not received antenatal steroids. In the control group, 2 patients had received antenatal steroids but 48 patients hadn't received steroids. Two groups did not significantly differ from each other for antenatal steroid use $(P=.221)$.

- $\quad$ Fluconazole recipient and control groups had no significant difference in receiving surfactant $(P=.6)$.

- The mean number days of mechanical ventilation was $3.7 \pm 7.82$ in the fluconazole group and $8.5 \pm 2.52$ in the control group; the mean days of mechanical ventilation was significantly higher in the patients of control group $(P=.002)$.

Duration of oxygen therapy was $7.86 \pm 11.33$ days in the group receiving IV fluconazole and $11.86 \pm 10.26$ days in the control group. Two groups were significantly different from each other regarding the number of days of oxygen therapy $(P=.04$; Figure 3$)$.

The mean number of hospital days in the group receiving fluconazole was $35.16 \pm 16.43$ days and in the control group was $43.03 \pm 19.07$ which has been significantly less in the group receiving fluconazole $(P=.011$; Figure 4$)$.

In the group receiving fluconazole, there were 9 deaths and in the control group, 15 patients died. The 2 groups were statistically different from each other $(P=.045)$. 


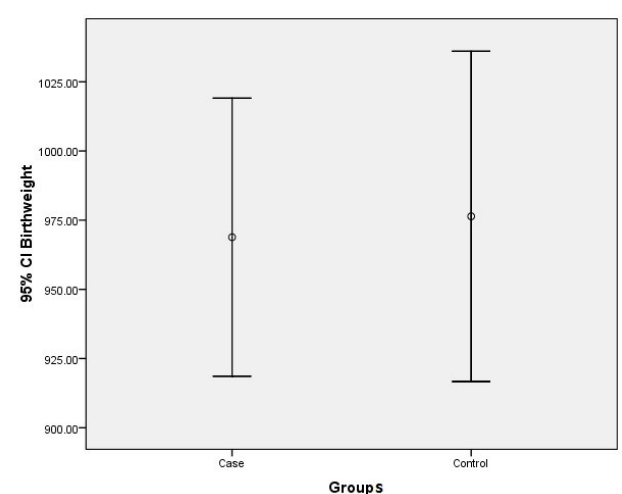

Figure 1. Neonates Birth Weight.

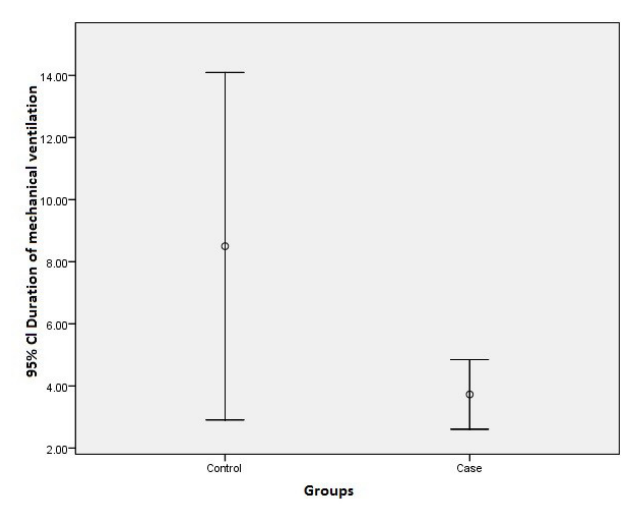

Figure 2. The Mean Gestational Age.

\section{Discussion}

Fungal infections such as infection with Candida species is one of the most dangerous opportunistic infections in neonates, especially in neonates with very low birth weight hospitalized in NICU leading to life-threatening infections. The first step in pathogenesis of this infection is the Candida colonization of the neonatal skin and gastrointestinal tract (7). Among the Candida species, Candida albicans is the most common species isolated from the infants; however, in the past decade, other types of this pathogen has been isolated from the infants, especially preterm infants with very low birth weight that can be related to the colonization of this pathogen in the vagina of infants' mothers, Candida carriage in health care workers, and birth of neonates with incomplete immune system. In general, these factors increase mortality and morbidity, especially among premature infants who spend a long time in the intensive care unit.

In different studies, the prophylactic antifungal effect was studied in preterm infants, but studies on this issue in our country have been very limited. In the present study, we examined the effect of intravenous fluconazole on the clinical outcome of preterm infants in a clinical trial conducted in Al-Zahra hospital, Tabriz, Iran. According to the results of present study, both case (receiving intravenous fluconazole) and control groups have been different in terms of the number of days of mechanical ventilation and oxygen requirement, hospitalization duration, and

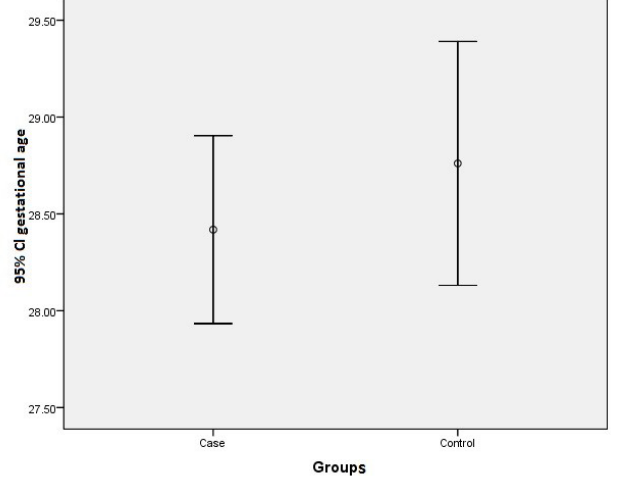

Figure 3. The Mean Duration of Mechanical Ventilation.

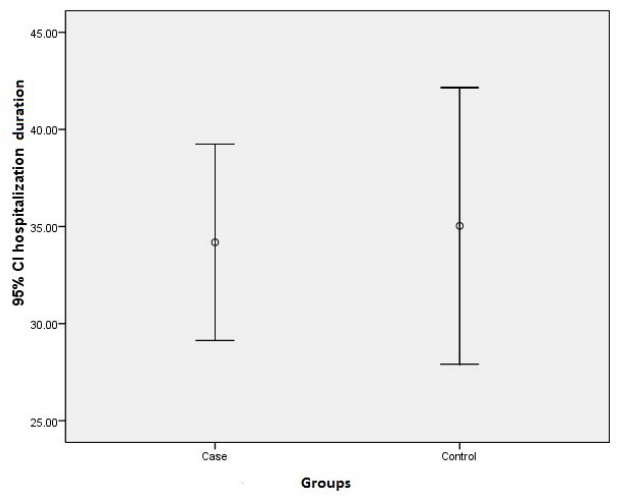

Figure 4. The Mean Days of Hospital Stay.

mortality rate; these variables were higher in the patients of control group that did not receive antifungal prophylaxis (8-10).

In the study of Castagnola et al (13) conducted on preterm infants, intravenous fluconazole reduced respiratory distress and the need for intubation and mechanical ventilation. In our study, the need for intubation and mechanical ventilation was significantly less in the case group which received the intravenous fluconazole. We can explain these differences with regard to the role of fungal factors on respiratory infections (11-13). Moreover, this study suggested that prophylaxis with antifungal drugs in several groups of preterm infants, including preterm neonates with very low birth weight infants, should be done routinely (11).

Despite inconsistent results of few studies with small sample size, prophylactic use of fluconazole in adults and children with hematologic malignancies and immunodeficiency diseases has been efficient for reducing the problems related to candidiasis $(8,12)$. Prophylactic use of fluconazole is suggested for some particular patients, including neutropenia patient with cancer, patients with HIV, solid-organ transplant recipients, patients with chronic granulomatous, patients with invasive Mycosis, and neonates with very low birth weight (15).

In our study, the duration of oxygen therapy in the group receiving intravenous fluconazole was $11.33 \pm 7.86$ days and in the control group was $11.86 \pm 10.26$ days, so there was a significant difference between two groups in terms 
of the number of days that there was the oxygen requirement. Furthermore, the mean duration of hospitalization was $35.16 \pm 16.43$ days in group receiving fluconazole but it was $43.03 \pm 19.07$ days in the control group showing it was significantly less in the fluconazole group compared with the control group $(P=.011)$; there were $9(20 \%)$ deaths in the fluconazole group while the number of deaths in the control group was 15 (30\%), the mortality rate was significantly higher in control group compared with the case group $(P=.045)$.

Weitkamp et al (12) conducted a study in order to evaluate antifungal treatment on hospitalization outcome in neonates with very low birth weight. They reported that antifungal drugs reduced the need for ventilation and respiratory distress among neonates receiving intravenous fluconazole that these results are compatible with the findings of our study.

Study of Kaufman et al (14) showed intravenous fluconazole is associated with less hospitalization duration and mortality rates, compatible with the finding of our study. In this study performed on 100 infants with very low birth weight, patients were divided into the case and control groups. Case group received fluconazole and the control group just received placebo; the results indicated the efficiency of fluconazole. In another study by Kaufman et al the effect of prophylactic fluconazole in preventing fungal infection in very low birth weight infants was studied. In this study, very low birth weight infants were treated by prophylactic intravenous fluconazole over a 4-year treatment period. Intravenous fluconazole could significantly prevent from Candida infections and deaths $(13,14)$. Therefore, the results of the recent study in which neonates receiving fluconazole had less mortality rate and better hospitalization outcome is comparable with the results of our study.

In another study, Aziz et al (15) examined 262 infants with very low birth weight allocated into two groups. In this study, 99 patient in the control group and 143 patients in the case group were examined. Although the patients in the control group in terms of birth weight, gestational week, and the start time of breast feeding were in a better position as compared with the case group, the study results indicated invasive fungal infection were less in the recipients of fluconazole, the infant mortality rate in this study was less in fluconazole recipients. Manzoni et al (11) in a study performed in 5 years, 465 infants with birth weight less than $1500 \mathrm{~g}$ were divided into 2 groups. One group received prophylactic fluconazole until 30 days after birth (for neonates less than $1000 \mathrm{~g}$, until 40 days after birth) and the control group did not receive fluconazole $(n=240)$. The effect of prophylactic fluconazole was examined on the neonatal outcomes. In this study, the total fungal colonization in the first group (which received fluconazole) was impressively less ( $24 \%$ vs $43.8 \%$ ). The mortality rate of neonates receiving prophylactic fluconazole was significantly less $(0 \%)$ compared with the control group (17\%) (11). In the study of Weitkamp et al (12), 86 patients were studied. Forty-four patients were in the placebo group and 42 patients in the group receiving prophylactic fluconazole. Nine cases of invasive fungal infection were seen in the fluconazole group with 3 deaths in infants, whereas there were no deaths in the placebo group. These findings are compatible with the findings of our study in which the mortality rate of neonates receiving intravenous fluconazole was significantly less compared with the control group (12).

\section{Conclusion}

The fungal infections are one of the most common causes of mortality and morbidity in preterm neonates and very low birth weight neonates. According to the results of present study on the prophylactic role of intravenous fluconazole in preterm neonates, it can be concluded that the need for mechanical ventilation, duration of hospitalization, and mortality rate of neonates receiving intravenous fluconazole is significantly less in comparison with controls without antifungal prophylaxis.

\section{Ethical issues}

The ethical issues were considered in this study.

\section{Conflict of interests}

The authors declare no conflict of interests.

\section{Finnacial support}

Not applicable.

\section{Acknowledgments}

We would like to thank all mothers who helped us in this study.

\section{References}

1. Makhoul IR, Sujov P, Smolkin T, Lusky A, Reichman B. Epidemiological, clinical, and microbiological characteristics of late-onset sepsis among very low birth weight infants in Israel: a national survey. Pediatrics. 2002;109(1):34-39.

2. Stoll BJ, Hansen N, Fanaroff AA, et al. Late-onset sepsis in very low birth weight neonates: the experience of the NICHD Neonatal Research Network. Pediatrics. 2002;110(2Pt):285-291.

3. Kossoff EH, Buescher ES, Karlowicz MG. Candidemia in a neonatal intensive care unit: trends during fifteen years and clinical features of 111 cases. Pediatr Infect Dis J. 1998;17(6):504-508.

4. Saiman L, Ludington E, Pfaller M, et al. Risk factors for candidemia in neonatal intensive care unit patients. The National Epidemiology of Mycosis Survey Study Group. Pediatr Infect Dis J. 2000;19(4):319-324.

5. Karlowicz MG, Rowen JL, Barnes-Eley ML, et al. The role of birth weight and gestational age in distinguishing extremely low birth weight infants at high risk of developing candidemia from infants at low risk: a multicenter study. Pediatr Res 2002;51:301A.

6. Baley JE, Kliegman RM, Boxerbaum B, Fanaroff AA. Fungal colonization in the very low birth weight 
infant. Pediatrics. 1986;78(2):225-232.

7. Huang YC, Li CC, Lin TY, et al. Association of fungal colonization and invasive disease in very low birth weight infants. Pediatr Infect Dis J. 1998;17(9):819822.

8. Pappu-Katikaneni LD, Rao KP, Banister E. Gastrointestinal colonization with yeast species and Candida septicemia in very low birth weight infants. Mycoses. 1990;33(1):20-23.

9. Rowen JL, Rench MA, Kozinetz CA, Adams JM Jr, Baker CJ. Endotracheal colonization with Candida enhances risk of systemic candidiasis in very low birth weight neonates. J Pediatr. 1994;124(5 Pt 1):789-794.

10. Saiman L, Ludington E, Dawson JD, et al. Risk factors for Candida species colonization of neonatal intensive care unit patients. Pediatr Infect Dis J. 2001;20(12):1119-1124.

11. Manzoni P, Stolfi I, Pugni L, et al. A multicenter, randomized trial of prophylactic fluconazole in preterm neonates. N Engl J Med. 2007;356(24):248395.

12. Weitkamp JH, Ozdas A, LaFleur B, Potts AL. Fluconazole prophylaxis for prevention of invasive fungal infections in targeted highest risk preterm infants limits exposure. J Perinatol. 2008;28(6):405411. doi:10.1038/sj.jp.7211914.

13. Castagnola E, Machetti M, Bucci B, Viscoli C. Antifungal prophylaxis with azole derivatives. Clin Microbiol Infect. 2004;10(Suppl 1):86-95.

14. Kaufman D, Boyle R, Hazen KC, Patrie JT, Robinson M, Donowitz LG. Fluconazole prophylaxis against fungal colonization and infection in preterm infants. N Engl J Med. 2001;345(23):1660-1666.

15. Aziz HA, Berrocal AM, Sisk RA, et al. Intraocular infections in the neonatal intensive care unit. Clin Ophthalmol 2012;6:733-737. doi:10.2147/OPTH. S26362.

Copyright () 2015 The Author(s); This is an open-access article distributed under the terms of the Creative Commons Attribution License (http://creativecommons.org/licenses/by/4.0), which permits unrestricted use, distribution, and reproduction in any medium, provided the original work is properly cited. 